\title{
Photoactivation of MDM2 Inhibitors: Controlling Protein-Protein Interaction with Light
}

\author{
Mickel J. Hansen, ${ }^{\dagger, \|}$ Femke M. Feringa, ${ }^{\ddagger}, \|$ Piermichele Kobauri, ${ }^{\dagger}$ Wiktor Szymanski, ${ }^{\dagger, \S_{(0)}}$ \\ René H. Medema, ${ }^{*}+$ and Ben L. Feringa ${ }^{*}+$ (i) \\ ${ }^{\dagger}$ Centre for Systems Chemistry, Stratingh Institute for Chemistry, University of Groningen, Nijenborgh 4, 9747 AG, Groningen, The \\ Netherlands \\ ${ }^{\ddagger}$ Oncode Institute, Netherlands Cancer Institute, Division of Cell Biology, Plesmanlaan 121, 1066 CX, Amsterdam, The Netherlands \\ ${ }^{\S}$ Department of Radiology, University Medical Center Groningen, University of Groningen, Hanzeplein 1, 9713 GZ Groningen, The
} Netherlands

Supporting Information

\begin{abstract}
Selectivity remains a major challenge in anticancer therapy, which potentially can be overcome by local activation of a cytotoxic drug. Such triggered activation can be obtained through modification of a drug with a photoremovable protecting group (PPG), and subsequent irradiation in the chosen place and time. Herein, the design, synthesis and biological evaluation is described of a photoactivatable MDM2 inhibitor, PPGidasanutlin, which exerts no functional effect on cellular outgrowth, but allows for the selective, noninvasive activation of antitumor properties upon irradiation visible light, demonstrating activation with micrometer, single cell precision. The generality of this method has been demonstrated by growth inhibition of multiple cancer cell lines showing p53 stabilization and subsequent growth inhibition effects upon irradiation. Light activation to regulate protein-protein interactions between MDM2 and p53 offers exciting opportunities to control a multitude of biological processes and has the potential to circumvent common selectivity issues in antitumor drug development.
\end{abstract}

$\mathrm{C}$ ancer is one of the major causes of death in the developed world. Long-standing drawbacks of cancer chemotherapy are its inherent toxicity and associated adverse effects. To fight these selectivity issues, targeting pathways that are exclusively needed for cancer cell survival have been explored. ${ }^{1-4}$ One way of controlling these cellular pathways is by interfering with cancer cell-specific protein-protein interactions (PPIs). Interestingly, by controlling PPIs, remote control of a specific protein can be achieved, which opens up new targeting strategies in anticancer treatment (see Figure 1).

The best known tumor suppressor protein, p53, is heavily involved in PPIs and plays an important role in cell-cycle control, apoptosis, DNA repair and cellular stress responses. ${ }^{5,6}$ Activation of p53 by various types of stress can drive cellular senescence, which is an irreversible cell-cycle arrest, to prevent potential transformation of the damaged cell. Utilizing its role in apoptosis and senescence, reactivation of the p53 signaling pathway remains a preeminent target for cancer treatment. ${ }^{7,8} \mathrm{~A}$

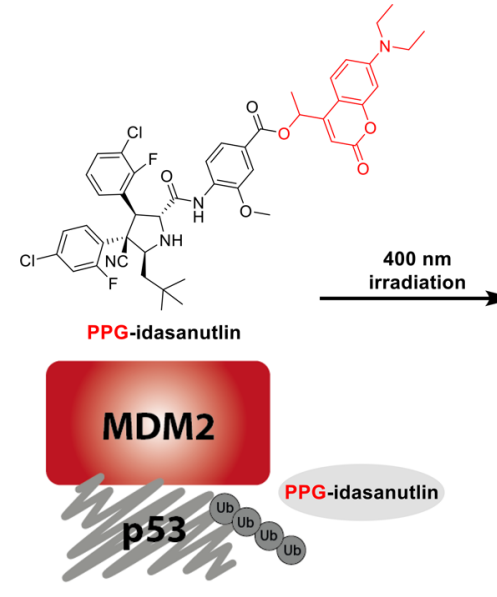

p53 degradation

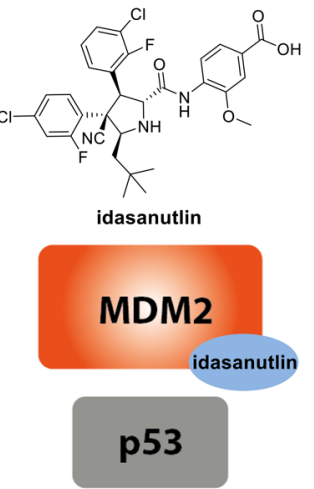

p53 stabilization

(Senescence)

(anti-tumor activity)
Figure 1. A schematic representation of the principles behind phototriggered p53 stabilization. Caged inhibitor (PPG-idasanutlin) is not able to inhibit the MDM2-p53 protein-protein interaction, which results in p53 ubiquitylation and degradation. Irradiation with $400 \mathrm{~nm}$ light releases the active inhibitor idasanutlin which prevents MDM2-p53 binding and as a consequence increases the p53 level, leading to senescence or cell death.

major concern in p53 reactivating therapies is its effect on normal cells, since upregulation of $\mathrm{p} 53$ protein expression by itself is sufficient to induce senescence or apoptosis in all cycling cells. ${ }^{9,10}$ Therefore, the selective activation of the p53 pathway in cancerous tissue is a key challenge, as it would greatly increase the potential success for therapeutical application.

One of the main repressors of p53 activity is the MDM2protein. MDM2 interacts with p53 to promote its ubiquitylation, making it a target for degradation by the proteasome

Received: May 9, 2018

Published: October 4, 2018 
<smiles>COc1cc(C(=O)O)ccc1NC(=O)[C@@H]1N[C@H](CC(C)(C)C)[C@@](C)(c2ccc(Cl)cc2F)[C@H]1c1cccc(Cl)c1F</smiles>

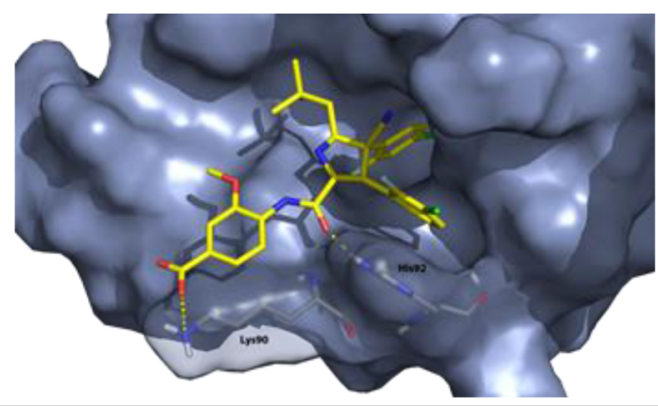

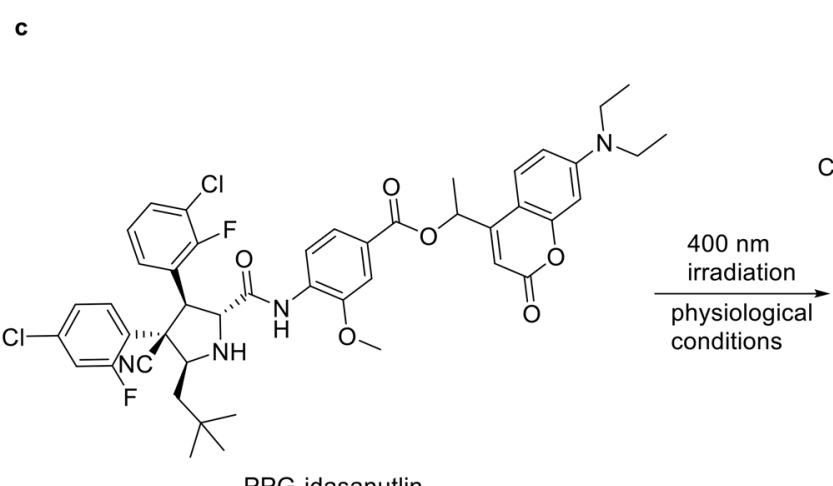

PPG-idasanutlin

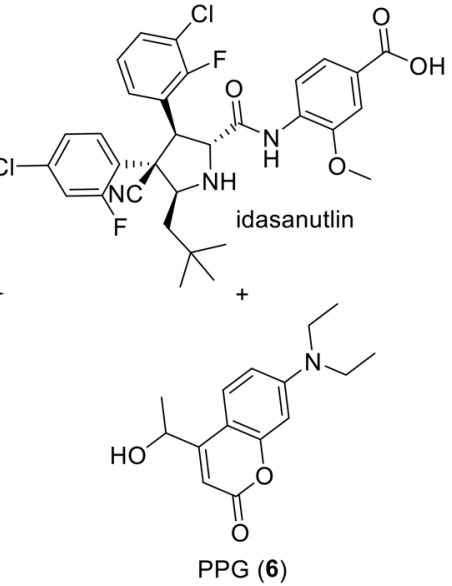
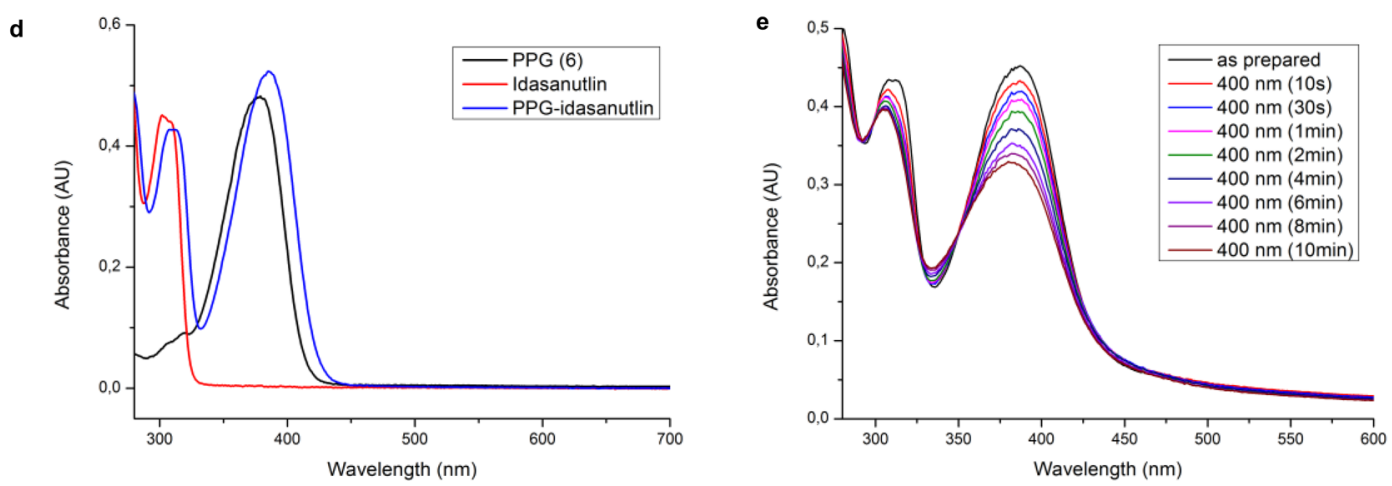

Figure 2. Strategy toward photocleavable nutlin derivatives. (a) Idasanutlin, a potent MDM2 inhibitor allowing the stabilization of p53 levels in tumor cells. (b) Molecular docking showcases the possible interaction with Lys90 as a potential site to alter the activity (PDB: 4JRG). ${ }^{29}$ (c) Irradiation of PPG-idasanutlin led to the formation of idasanutlin and PPG(6) as the sole products. (d) Absorption spectra of PPG-idasanutlin, idasanutlin and PPG(6) in buffer. ${ }^{30}$ (e) UV-vis spectra of PPG-idasanutlin upon exposure to $400 \mathrm{~nm}$ light showing a clean photochemical conversion (isosbestic point at $350 \mathrm{~nm}$ ) to the desired products, see SI for detailed UPLC-MS studies.

(Figure 1). ${ }^{11-13}$ The regulatory PPI between p53 and MDM2 makes the latter an interesting target in anticancer drug development. Recently, a class of MDM2 inhibitors (nutlins) have been developed allowing the selective activation of the tumor suppressing p53 pathway (Figure 1; structure of idasanutlin shown). ${ }^{14-16}$ Nutlins bind to the p53-binding site of MDM2, inhibiting proteolytic breakdown of p53, resulting in the stabilization of $\mathrm{p} 53$ which arrests rapid cell division and can induce senescence.

To ultimately increase the selectivity of such MDM2 inhibitors and to utilize them as a research tool to investigate MDM2-p53 interactions, photopharmacological strategies ${ }^{17,18}$ can be applied in which a drug is modified with a photoswitch, ${ }^{17,18}$ or photoremovable protecting group. ${ }^{19-21}$ Masking of a functional group in a pharmacophore with a photoremovable protecting group allows its selective light- triggered activation. Ideally, the photoprotected drug is inactive, while after photodeprotection the active drug is liberated, taking advantage of the noninvasive nature of light. $^{22,23}$

Herein, we describe the design, synthesis and biological evaluation of a photoactivatable MDM2 inhibitor PPGidasanutlin. The principle of phototriggered p53 stabilization is shown in Figure 1. The caged inhibitor (PPG-idasanutlin) is not capable of blocking the MDM2-p53 protein-protein interaction, resulting in p53 degradation. Photochemical release of idasanutlin prevents MDM2-p53 binding, triggering senescence or cell death. The system described herein allows, for the first time, the selective light-activation of tumorarresting p53 in living cells.

Our molecular design was based on a recently developed MDM2 inhibitor, idasanutlin (see Figure 2a), which showed 


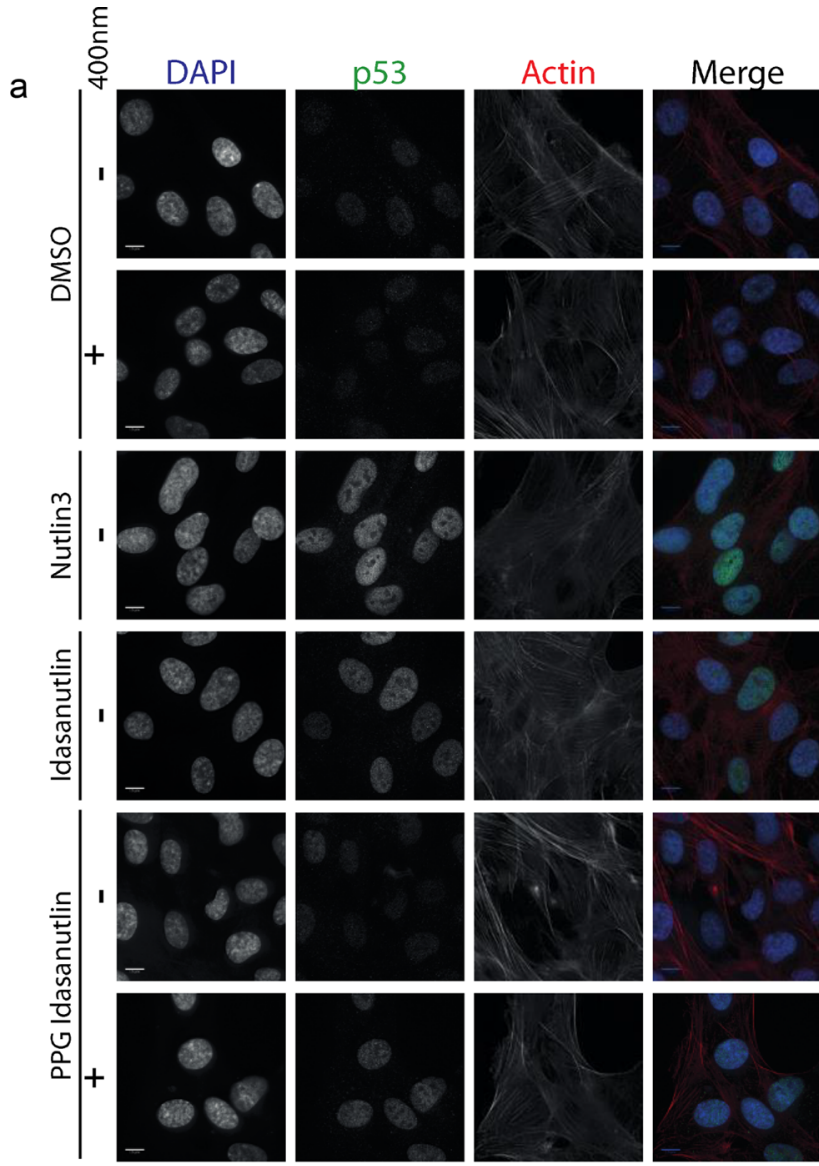

b

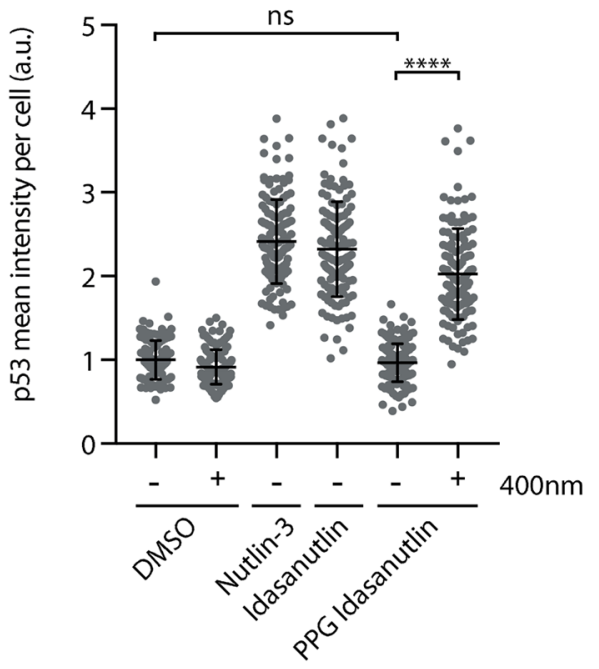

C

d

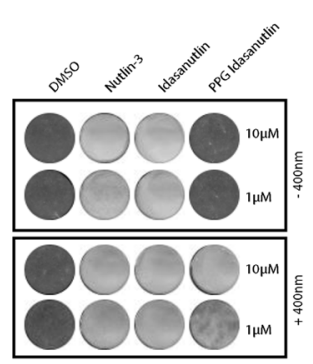

e
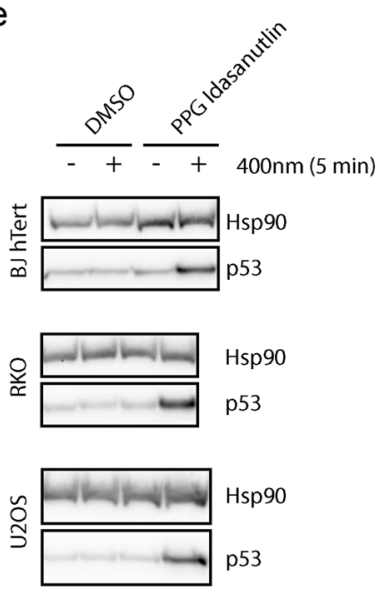

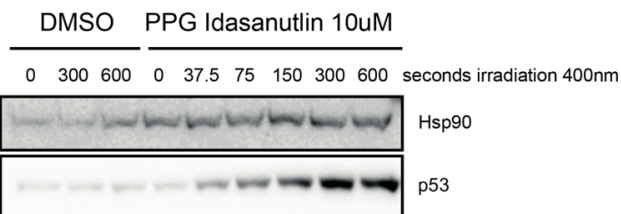

f
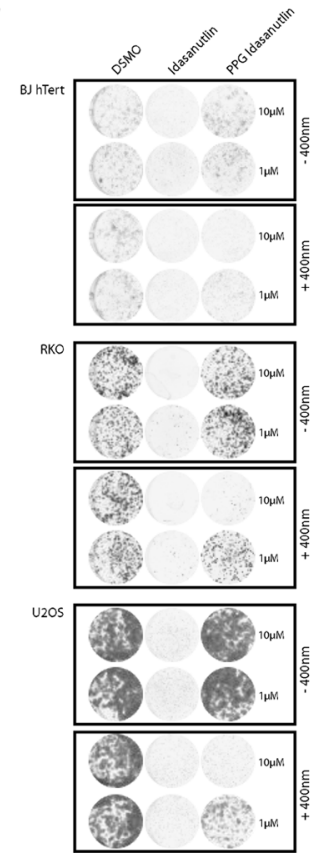

Figure 3. Functional p53 induction upon $\lambda=400 \mathrm{~nm}$ irradiation in PPG-idasanutlin treated cells. (a) RPE-1 cells were treated with indicated compounds (all $10 \mu \mathrm{M}$ final) and fixed $4 \mathrm{~h}$ after $5 \mathrm{~min}(\mp 400 \mathrm{~nm})$ irradiation. ${ }^{34}$ (b) Quantification of the mean p53 intensity per nucleus in cells treated as in $(\mathrm{a}) .^{35}$ (c) Representative Western blot showing p53 protein levels in cells $4 \mathrm{~h}$ after addition of DMSO or PPG-idasanutlin and irradiation for indicated time periods. Hsp90 is used as a loading control. (d) Selective outgrowth disadvantage in RPE-1 cells 6 days after PPGidasanutlin treatment $+400 \mathrm{~nm}$ irradiation for $5 \mathrm{~min}$. (e) Representative Western blot showing p53 protein levels in three cell lines (U2OS, RKO, BJhTert) $4 \mathrm{~h}$ after indicated treatments. (f) Selective outgrowth inhibition in indicated cell lines 6 days after PPG-idasanutlin treatment $+400 \mathrm{~nm}$ irradiation for $5 \mathrm{~min}^{36}$ 
a

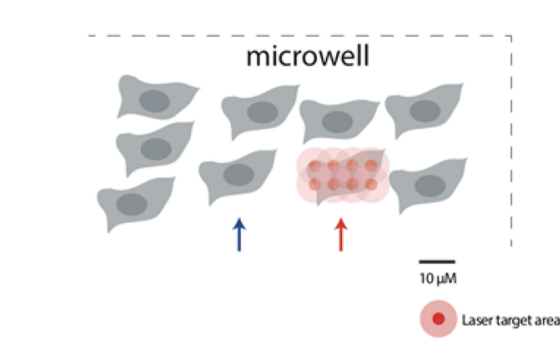

$\rightarrow$ Example cell not hit by the laser

$\rightarrow$ Example cell hit by the laser ( 0,1 sec pulse per position)

b

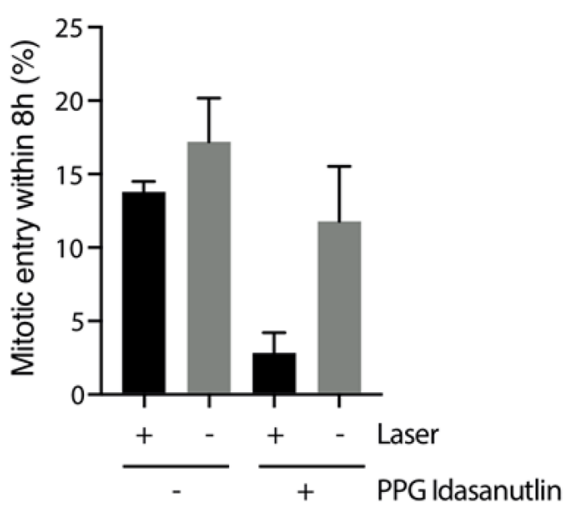

d $\quad$ - PPG Idasanutlin
C + PPG Idasanutlin

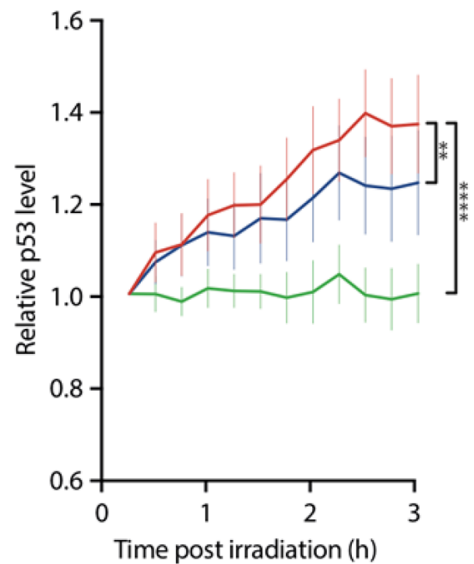

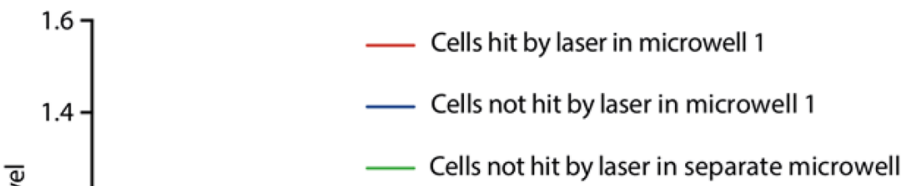

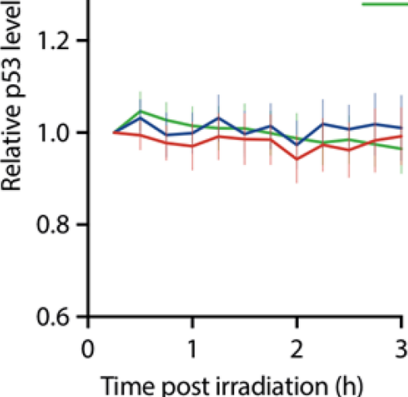

Figure 4. Spatiotemporal control of PPG-idasanutlin. (a) Schematic representation of microwell setup for laser irradiation of individual RPE-1 cells to activate PPG-idasanutlin. Laser target area (represented by red circle) for single pulse $(0.1 \mathrm{~s}$ irradiation at $5 \mu \mathrm{m}$ interspaced position) indicated with scale. Individual irradiated cells followed by measuring nuclear p53-venus levels (fluorescence) every 15 min for $3 \mathrm{~h}$ after laser irradiation. Approximately 200 cells in each microwell. (b) Percentage of cells that divide within $8 \mathrm{~h}$ after indicated treatments. ${ }^{38}$ (c,d) p53-venus fluorescent signal in individual RPE-1 cells tracked over time after indicated treatments as represented in (a). ${ }^{39}$

high potency, moderate selectivity and good bioavailability. ${ }^{7,14,16}$ From limited SAR studies, ${ }^{29}$ it can be concluded that the $m$-methoxybenzoic acid group plays a potential role in binding affinity, cellular potency/stability and pharmacokinetic properties (see Figure 2a, marked red). Synthetic modification of the $m$-methoxybenzoic acid potentially renders the nutlin derivative inactive. The possibility to alter the activity of idasanutlin by masking of this functional group was further established by docking studies suggesting that a potential interaction with Lys 90 is prevented in the protected compound (Figure $2 \mathrm{~b}$ and SI for computational details).

Encouraged by these preliminary docking studies, we designed a photoactivatable idasanutlin (PPG-idasanutlin) which would potentially show a difference in activity between the protected and photodeprotected forms. We selected the coumarin scaffold as the PPG of choice, which is known to allow for a fast deprotection with biocompatible visible light $(\lambda$ $>400 \mathrm{~nm}$ ) without the generation of toxic side products. The hydroxymethylcoumarin was preferred over the normal hydroxycoumarin because of its improved hydrolytic stability and increased rate of photocleavage. ${ }^{21,24,25}$

Following the synthesis of the desired PPG-idasanutlin (see SI for details), ${ }^{21,26-28}$ we investigated its photochemical behavior under physiological conditions. From UV-vis spectroscopy and UPLC-MS measurements in aqueous buffer at $\mathrm{pH}=7.0$, photodeprotection with $\lambda=400 \mathrm{~nm}$ light was observed, showing solely the formation of idasanutlin and hydroxycoumarin. The rate of photocleavage proved to be high, allowing the major photorelease of idasanutlin within 5 min of irradiation, with a $0.1 \%$ quantum yield. ${ }^{22}$ Moreover, no significant spontaneous hydrolysis of PPG-idasanutlin for $>24$ $\mathrm{h}$ in buffer at room temperature was observed. This allows the application of PPG-idasanutlin under physiological assay conditions using short irradiation times with biocompatible visible (>400 $\mathrm{nm}$ ) light.

Next, the biological activity of PPG-idasanutlin was investigated. Initial studies aimed at confirming a difference in p53 activation upon $\lambda=400 \mathrm{~nm}$ light exposure after addition of the protected idasanutlin derivative (PPGidasanutlin). Nontransformed, p53-proficient retinal pigment epithelial cells (RPE-1) were treated with DMSO (control), nutlin-3, idasanutlin or PPG-idasanutlin followed by $\mp$ irradiation with $400 \mathrm{~nm}$ light (Figure 3). Immunofluorescent staining revealed a significant increase in nuclear $\mathrm{p} 53$ protein levels in cells $4 \mathrm{~h}$ after addition of nutlin-3 or idasanutlin, regardless of the irradiation with $400 \mathrm{~nm}$ light. Importantly, treatment with PPG-idasanutlin only resulted in a significant increase in p53 protein level when these cells were irradiated with $400 \mathrm{~nm}$ light (photorelease of idasanutlin, see Figure $3 a, b){ }^{31}$ To examine the level of control over the dose- 
response of idasanutlin (employing PPG-idasanutlin), p53 protein levels in RPE-1 cells were determined by immunostaining after both increasing duration of $400 \mathrm{~nm}$ light irradiation and varying doses of PPG-idasanutlin (Figure $3 \mathrm{c}$ and Figure S16). The clear dose-response dependent accumulation of p53 protein shows the highly effective light controllable dose responsiveness of the biological effect using PPG-idasanutlin (Figure 3c).

Subsequently, the functional ability to photocontrol growth of rapidly dividing cells was investigated. Colony outgrowth of RPE-1 cells treated with PPG-idasanutlin was selectively blocked after irradiation with $400 \mathrm{~nm}$, while irradiation did not perturb outgrowth of DMSO treated cells (Figure 3d). ${ }^{32}$ This showcases the use of $400 \mathrm{~nm}$ light in living systems as a valid approach to photocontrol biological function. It should be emphasized that in the outgrowth experiment seen in Figure $3 \mathrm{~d}$, treatment of cells with PPG-idasanutlin without $400 \mathrm{~nm}$ irradiation did not show any growth inhibition, confirming the lack of inherent activity of the protected idasanutlin. In other words PPG-idasanutlin has no functional effect on p53 stabilization nor compromises cellular outgrowth. ${ }^{33}$

To verify whether (re)activation of p53 by our light controllable PPG-idasanutlin is more generally applicable and not dependent on the nontransformed RPE-1 cells used in these experiments, additional nontransformed (BJ-hTert) and tumor (RKO (colon carcinoma), U2OS (osteosarcoma)) cell lines were included for follow-up analysis. Selective stabilization of p53, after treatment with PPG-idasanutlin and light irradiation, was observed in all cell lines tested (Figure $3 \mathrm{e}$ ). The light-controlled p53 activation invariably led to a dramatic reduction in cellular outgrowth (Figure $3 \mathrm{f}$ ) proving the possibility to control tumor cellular growth using PPGidasanutlin and light.

To demonstrate the spatiotemporal control of the designed system, we sought to investigate the selective enhancement of p53 levels in individual RPE-1 cells within a cell population using light irradiation. ${ }^{37}$ Using RPE-1 cells that stably expressed a venus-tagged version of p53 (p53-venus), p53 protein accumulation could be tracked (see SI for details) with high time-resolution in individual cells by live-cell microscopy. $\mathrm{RPE}$ p53-venus cells were grown in $620 \mu \mathrm{m}$ wide microwells and a $405 \mathrm{~nm}$ laser was used to irradiate individual cells in the colony with a single $0.1 \mathrm{~s}$ pulse at $5 \mu \mathrm{m}$ inter spaced positions to acquire micrometer precision (Figure 4a). To determine the functionality of the high spatiotemporal control obtained in this setup, cell cycle progression was monitored in single cells following laser activation of PPG-idasanutlin (photorelease of idasanutlin). Functional p53 activation will halt cell division, causing fewer cells to pass through mitosis. ${ }^{9}$ Indeed, the percentage of cells that divide within $8 \mathrm{~h}$ after the indicated treatment strongly drops in cells that were irradiated after treatment with PPG-idasanutlin (Figure 4b). This shows that a specific cellular fate can be induced at single-cell resolution by laser irradiation as presented in Figure 4a. Quantification of the nuclear p53-venus signal at 15 min intervals in single cells treated with PPG-idasanutlin revealed the selective stabilization of p53 protein following irradiation with the $405 \mathrm{~nm}$ laser (Figure 4c). A significantly lesser extent of p53 stabilization was detectable in neighboring cells that were not irradiated by the $405 \mathrm{~nm}$ laser (Figure 4c). The limited stabilization of the nonirradiated neighboring cells is most likely explained by diffusion of activated PPG-idasanutlin (idasanutlin) within the excess of liquid cell culture medium in this $2 \mathrm{D}$ cell culture setup. In contrast, p53 stabilization was completely absent in nonirradiated cells from adjacent wells at micrometer distance, where diffusion could not take place. In addition, p53 levels did not increase due to laser-induced damage to the cells, since p53 levels were unaltered in cells following an identical irradiation protocol in absence of PPG-idasanutlin (Figure 4d). Together these results show the selective activation of PPGidasanutlin resulting in the release of idasanutlin, using an extremely short $(0.1 \mathrm{~s})$ pulse of $405 \mathrm{~nm}$ laser irradiation at micrometer, single-cell resolution, which offers promising opportunities for future studies using PPG-idasanutlin in 3D settings like (tumor) tissue.

In summary, the PPG-idasanutlin reported herein allows the photocontrol of protein-protein interactions and their functional outcome. Stabilization of p53 and consequent cell growth arrest could be obtained by MDM2 inhibition upon photoactivation with biocompatible $400 \mathrm{~nm}$ light. Excitingly, spatiotemporal control was achieved with microsecond irradiation at micrometer, single-cell resolution. This constitutes, to the best of our knowledge, the first system that externally and indirectly controls p53 levels with light. Next to a promising concept toward selective anticancer therapy, the designed system can also function as a molecular tool to investigate MDM2-p53 interactions as well as selectively interfere with the numerous cellular processes regulated by p53.

\section{ASSOCIATED CONTENT}

\section{S Supporting Information}

The Supporting Information is available free of charge on the ACS Publications website at DOI: 10.1021/jacs.8b04870.

Materials and methods, synthetic schemes, experimental procedures, photochemical data, NMR spectra and HRMS of new compounds (PDF)

\section{AUTHOR INFORMATION}

\section{Corresponding Authors}

*r.medema@nki.nl

*b.l.feringa@rug.nl

ORCID

Wiktor Szymanski: 0000-0002-9754-9248

Ben L. Feringa: 0000-0003-0588-8435

\section{Author Contributions}

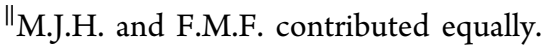

\section{Notes}

The authors declare no competing financial interest.

\section{ACKNOWLEDGMENTS}

This work was financially supported by The Netherlands Organization for Scientific Research (NWO-CW, Top grant to B.L.F. and NWO VIDI grant no. 723.014 .001 for W.S.), the Dutch Cancer Foundation (KWF; NKI 2014-6787), the Royal Netherlands Academy of Arts and Sciences (KNAW), the Ministry of Education, Culture and Science (Gravitation programme 024.001.035), and the European Research Council (Advanced Investigator Grant no. 694345 to B.L.F.). We thank all Medema, Rowland, Jacobs and Feringa lab members for helpful discussions on this study. We kindly thank Dr. Galit Lahav for sharing the RPE-1 p53-venus cell line. 


\section{REFERENCES}

(1) Stewart, B.; Wild, C. World Cancer Report 2014 http:// publications.iarc.fr/Non-Series-Publications/World-Cancer-Reports/ World-Cancer-Report-2014 (accessed Jun 16, 2017).

(2) Moslehi, J. J. Cardiovascular Toxic Effects of Targeted Cancer Therapies. N. Engl. J. Med. 2016, 375, 1457-1467.

(3) Bild, A. H.; Yao, G.; Chang, J. T.; Wang, Q.; Potti, A.; Chasse, D.; Joshi, M.-B.; Harpole, D.; Lancaster, J. M.; Berchuck, A.; Olson, J. A., Jr; Marks, J. R.; Dressman, H. K.; West, M.; Nevins, J. R. Oncogenic Pathway Signatures in Human Cancers as a Guide to Targeted Therapies. Nature 2006, 439, 353-357.

(4) van 't Veer, L. J.; Bernards, R. Enabling Personalized Cancer Medicine through Analysis of Gene-Expression Patterns. Nature 2008, 452, 564-570.

(5) Vogelstein, B.; Kinzler, K. W. P53 Function and Dysfunction. Cell 1992, 70, 523-526.

(6) Kastenhuber, E. R.; Lowe, S. W. Putting P53 in Context. Cell 2017, 170, 1062-1078.

(7) Lakoma, A.; Barbieri, E.; Agarwal, S.; Jackson, J.; Chen, Z.; Kim, Y.; Mcvay, M.; Shohet, J.; Kim, E. The MDM2 Small-Molecule Inhibitor RG7388 Leads to Potent Tumor Inhibition in P53 WildType Neuroblastoma. Cell Death Discovery 2015, 1, 15026.

(8) Brown, C. J.; Lain, S.; Verma, C. S.; Fersht, A. R.; Lane, D. P. Awakening Guardian Angels: Drugging the P53 Pathway. Nat. Rev. Cancer 2009, 9, 862-873.

(9) Krenning, L.; Feringa, F. M.; Shaltiel, I. A.; Van Den Berg, J.; Medema, R. H. Transient Activation of P53 in G2 Phase Is Sufficient to Induce Senescence. Mol. Cell 2014, 55, 59-72.

(10) Burgess, A.; Chia, K. M.; Haupt, S.; Thomas, D.; Haupt, Y.; Lim, E. Clinical Overview of MDM2/X-Targeted Therapies. Front. Oncol. 2016, 6, 7.

(11) Haupt, Y.; Maya, R.; Kazaz, A.; Oren, M. Mdm2 Promotes the Rapid Degradation of P53. Nature 1997, 387, 296-299.

(12) Honda, R.; Tanaka, H.; Yasuda, H. Oncoprotein MDM2 Is a Ubiquitin Ligase E3 for Tumor Suppressor P53. FEBS Lett. 1997, 420, 25-27.

(13) Kubbutat, M. H. G.; Jones, S. N.; Vousden, K. H. Regulation of P53 Stability by Mdm2. Nature 1997, 387, 299-303.

(14) Vassilev, L. T.; Vu, B. T.; Graves, B.; Carvajal, D.; Podlaski, F.; Filipovic, Z.; Kong, N.; Kammlott, U.; Lukacs, C.; Klein, C.; Fotouhi, N.; Liu, E. A. Vivo Activation of the P53 Pathway by Small-Molecule Antagonists of MDM2. Science 2004, 303, 844-848.

(15) Harris, S. L.; Levine, A. J. The P53 Pathway: Positive and Negative Feedback Loops. Oncogene 2005, 24, 2899-2908.

(16) Ray-Coquard, I.; Blay, J.; Italiano, A.; Le Cesne, A.; Penel, N.; Zhi, J.; Heil, F.; Rueger, R.; Graves, B.; Ding, M.; Geho, D.; Middleton, S. A.; Vassilev, L. T.; Nichols, G. L.; Bui, B. N. Effect of the MDM2 Antagonist RG7112 on the P53 Pathway in Patients with MDM2-Amplified, Well-Differentiated or Dedifferentiated Liposarcoma: An Exploratory Proof-of-Mechanism Study. Lancet Oncol. 2012, 13, 1133-1140.

(17) Lerch, M. M.; Hansen, M. J.; van Dam, G. M.; Szymanski, W.; Feringa, B. L. Emerging Targets in Photopharmacology. Angew. Chem., Int. Ed. 2016, 55, 10978-10999.

(18) Broichhagen, J.; Frank, J. A.; Trauner, D. A Roadmap to Success in Photopharmacology. Acc. Chem. Res. 2015, 48, 1947-1960.

(19) Velema, W. A.; van der Berg, J. P.; Szymanski, W.; Driessen, A. J. M.; Feringa, B. L. Orthogonal Control of Antibacterial Activity with Light. ACS Chem. Biol. 2014, 9, 1969-1974.

(20) Stanton-Humphreys, M. N.; Taylor, R. D. T.; McDougall, C.; Hart, M. L.; Brown, C. T. a.; Emptage, N. J.; Conway, S. J. Wavelength-Orthogonal Photolysis of Neurotransmitters in Vitro. Chem. Commun. 2012, 48, 657-659.

(21) Gandioso, A.; Cano, M.; Massaguer, A.; Marchan, V. A Green Light-Triggerable RGD Peptide for Photocontrolled Targeted Drug Delivery: Synthesis and Photolysis Studies. J. Org. Chem. 2016, 81, $11556-11564$.

(22) Klán, P.; Šolomek, T.; Bochet, C. G.; Blanc, A.; Givens, R.; Rubina, M.; Popik, V.; Kostikov, A.; Wirz, J. Photoremovable
Protecting Groups in Chemistry and Biology: Reaction Mechanisms and Efficacy. Chem. Rev. 2013, 113, 119-191.

(23) Hansen, M. J.; Velema, W. A.; Lerch, M. M.; Szymanski, W.; Feringa, B. L. Wavelength-Selective Cleavage of Photoprotecting Groups: Strategies and Applications in Dynamic Systems. Chem. Soc. Rev. 2015, 44, 3358-3377.

(24) Weis, S.; Shafiq, Z.; Gropeanu, R. A.; del Campo, A. Ethyl Substituted Coumarin-4-Yl Derivatives as Photoremovable Protecting Groups for Amino Acids with Improved Stability for SPPS. J. Photochem. Photobiol., A 2012, 241, 52-57.

(25) Gandioso, A.; Contreras, S.; Melnyk, I.; Oliva, J.; Nonell, S.; Velasco, D.; García-Amorós, J.; Marchán, V. Development of Green/ Red-Absorbing Chromophores Based on a Coumarin Scaffold That Are Useful as Caging Groups. J. Org. Chem. 2017, 82, 5398-5408.

(26) Shu, L.; Gu, C.; Fishlock, D.; Li, Z. Practical Synthesis of MDM2 Antagonist RG7388. Part 1: A Cu(II)-Catalyzed Asymmetric [3 + 2] Cycloaddition. Org. Process Res. Dev. 2016, 20, 2050-2056.

(27) Rimmler, G.; Alker, A.; Bosco, M.; Diodone, R.; Fishlock, D.; Hildbrand, S.; Kuhn, B.; Moessner, C.; Peters, C.; Rege, P. D.; Schantz, M. Practical Synthesis of MDM2 Antagonist RG7388. Part 2: Development of the $\mathrm{Cu}(\mathrm{I})$ Catalyzed $[3+2]$ Asymmetric Cycloaddition Process for the Manufacture of Idasanutlin. Org. Process Res. Dev. 2016, 20, 2057-2066.

(28) Tsakos, M.; Schaffert, E. S.; Clement, L. L.; Villadsen, N. L.; Poulsen, T. B. Ester Coupling Reactions - an Enduring Challenge in the Chemical Synthesis of Bioactive Natural Products. Nat. Prod. Rep. 2015, 32, 605-632.

(29) Ding, Q.; Zhang, Z.; Liu, J.-J.; Jiang, N.; Zhang, J.; Ross, T. M.; Chu, X.-J.; Bartkovitz, D.; Podlaski, F.; Janson, C.; Tovar, C.; Filipovic, Z. M.; Higgins, B.; Glenn, K.; Packman, K.; Vassilev, L. T.; Graves, B. Discovery of RG7388, a Potent and Selective P53-MDM2 Inhibitor in Clinical Development. J. Med. Chem. 2013, 56, 59795983.

(30) Buffer contains: TRIS, BIS-TRIS, MES, NaOAc, $25 \mathrm{mM}$ each, $\mathrm{pH}=$ 7.0. PPG-idasanutlin at $20 \mu \mathrm{M}$ concentration.

(31) This increase is not a result of a stress response potentially activated by irradiation of the cells with $400 \mathrm{~nm}$ light, since DMSO control treated cells did not show any increase in p53 expression following irradiation (Figure 3a,b).

(32) PPG (6), the photoproduct after photodeprotection, was also tested and proved to be biologically inactive (see Figure S15 for details).

(33) Fluorescence microscopy indicates that cellular uptake of protected idasanultin (PPG-idasanutlin) is not prevented.

(34) Anti-p53 staining indicates p53 protein expression in the nucleus. DNA stained by DAPI and actin staining shows the cytoskeleton of the cell.

(35) Error bars represent mean + sd. $* * * * p<0.0001$ (unpaired $t$ test). Dots represent individual cells, $n>125$ cells per condition combined from 2 independent experiments.

(36) In all the experiments $<1 \%$ DMSO was used.

(37) For photoactivatable probes for single cell imaging, see: Tran, M. N.; Rarig, R-A. F.; Chenoweth, D. M. Synthesis and Properties of Lysosome-specific Photoactivatable Probes for Live-Cell Imaging. Chem. Sci. 2015, 6, 4508-4512, and references therein. For control of cellular peptide uptake using an azobenzene switch, see: Prestel, A.; Möller, H. M. Spatio-temporal Control of Cellular Uptake Achieved by Photoswitchable Cell-Penetrating Peptides. Chem. Commun. 2016, 52, 701-704.

(38) Bar graph shows mean + sem of three independent experiments. Error bars indicate $95 \%$ confidence intervals.

(39) Line-graphs represent mean of individual cells. $n>42$ cells per condition pooled from three independent experiments. $* * * p<0,005$, $* * * * p<0,0001$ significance in 2-way ANOVA interaction score. 